\title{
Human Papilloma Virus (HPV) and Genital Cancer
}

\author{
C. Rodríguez-Cerdeira ${ }^{*}, 1$, R. Alcántara ${ }^{2}$, A. Guerra-Tapia ${ }^{3}$, J. Escalas $^{4}$ and A. Alba ${ }^{5}$ \\ ${ }^{I}$ Dermatology Department, CHUVI \& University of Vigo, Vigo, Spain \\ ${ }^{2}$ Dermatology Department, Instituto Dermatologico, Santo Domingo, R. Domicana \\ ${ }^{3}$ Dermatology Department, Hospital Doce de Octubre, Madrid, Spain \\ ${ }^{4}$ Dermatology Department, Hospital Son Dureta, Palma de Mallorca, Spain \\ ${ }^{5}$ Centro de Estudios Celulares y Moleculares, Lugo, Spain
}

\begin{abstract}
Epidemiological relationships were established more than 100 years ago between precursory lesions of cervical cancer and risky sexual behaviour. For various decades medicine has, unsuccessfully, tried to find the relationships between the agents responsible for sexually transmitted infections with this neoplasia.

Thus, epidemiological studies supported by liquid cytological and molecular techniques have confirmed the role of certain strains of human papilloma virus (HPV) in the development of cervical, vulvar and vaginal cancer. It has been shown that, in an international series using high PCR, 90.7\% of cervical-uterine carcinomas have DNA from HPV, and, 100\% of the cases have been confirmed with exhaustive histological examinations, and in the majority of the intraepithelial lesions of the lower genital tract.

Persistent infection with high-risk HPV-types is associated with genital cancers. Smoking and HIV infection have consistently been associated with longer duration of HPV infection and risk for genital cancer.

We present the most important clinical manifestations of HPV infection within the spectrum of epithelial anomalies, considered as precursors or pre-colonizers of genital cancer and an update of their possible treatments.
\end{abstract}

Keywords: HPV, high risk HPV, genital cancer.

\section{INTRODUCTION}

Human papilloma virus (HPV) is one of the most common sexually transmitted diseases. At present more than 120 strains of HPV are recognised, each of which shows its own particular tropism for specific anatomical sites. Skin infections of the mucous membranes of the oral, respiratory and anogenital tracts are the most common. The International Agency for Cancer Research (IACR), which belongs to the WHO, classifies HPV types 16 and 18 as carcinogenic and types 31 and 33 as probably carcinogenic [1]. More recently, based on data from 11 studies carried out in various countries, in which the relationship between the cervix and HPV have been studied, 15 high-risk oncological viral strains have been identified (HR-HPV) $(16,18,31,33,35$, $39,45,51,52,56,58,59,68,73$ y 82 ); strains 26,53 and 66 have been classified as possible high-risk, and strains 6,11 , 40, 42, 43, 44, 54, 61, 70, 72, 81 and CP6108 have been classified as low-risk (LR-HPV) [2].

Over the last 35 years we have observed an important increase in the prevalence of HPV infection, both in its clinical forms or condylomas, and its sub-clinical expression,

*Address correspondence to this author at the Department of Dermatology, CHUVI, Meixoeiro Hospital \& University of Vigo, 36200 Vigo (Galicia), Spain; Tel: 00-34-600536114; Fax: 00-34-986-276416;

E-mail: aristoteles_cerdeira@yahoo.es; carmen.rodriguez.cerdeira@yahoo.es identified by cytological changes, colposcopy and/or vulvoscopy and/or vaginoscopy. Clinical forms, generally caused by low oncological risk HPV strains $(6,11)$, are generally benign. Sub-clinical forms include both benign lesions and lesions with pre-malignant potential, and are generally caused by high oncological risk HPV strains (16 and 18). In a recent study carried out by the IACR group in 13 areas in 11 countries, among which Spain was included, a high prevalence of HPV, both in Europe and Sub-Saharan Africa was found. It was, furthermore, observed that HPV16 infection was more frequent in European women. Finally, great heterogeneity was observed with respect to HPV strains in the study groups [3-5].

Thus, epidemiological studies supported by molecular techniques and liquid cytology have confirmed the incidental role of certain strains of HPV in the development of cervical, vulvar and vaginal cancer. An international series with high sensitivity PCR has proven that HPV DNA is present in $90.7 \%$ of cervical-uterine carcinomas and is present in $100 \%$ of cases confirmed by exhaustive histological examination. This also occurs in the majority of intraepithelial lesions of the lower genital tract (LGT). Strain 16 showed the highest prevalence in half the cases, followed by strain 18, which was involved in $12 \%$ of the cases. The sequence which, possibly, exists in the high grade lesions is associated with infection by high-risk HPV strains, albeit not exclusively. Co-infections by HPV-16 and HPV-58 appear to be especially dangerous [6]. 
At present it is accepted that cervical cancer is a sexually transmitted disease (STD). The WHO considers that STD constitutes the second most important cause of disease in young women in developing countries, and the main cause of infertility, disability and death in the world. Infection by HPV may be expressed in clinical, sub-clinical or latent form. HPV infection is increasing alarmingly. The ability of the virus to establish sub-clinical infection and its association with malignancy of the lower feminine tract makes the evaluation of statistical results all the more worrying.

Continuous advances with important practical implications have emerged since the confirmation of the viral aetiology of genital cancer [7]. Therefore, bringing up to date certain knowledge on genital infection by HPV is justified. This is based on the following novelties:

1. Knowledge of the development of HPV infection and precursory lesions in cancer of the LGT and anus, and its implication in these cancers.

2. Study of the associated risks of certain genotypes of HPV and their oncological roles.

3. Investigation of coexisting factors and their role in the development of cancer.

4. Cost-benefit study of screening efficiency as a tool for detecting HPV infection in the general public.

5. Improvement and refinement of the diagnostic methods; as well as advances in colposcopic inspection of the lesions, and the possibility to use digital colposcopy for the follow-up of the low-grade lesions and the introduction of the vulvar biopsy as a routine technique.

6. Study of the efficiency of various treatments for HPV infection. Development of new vaccines.

Thus, it is fitting that we are provided with updated documentation, based on evidence, which reviews the present state of knowledge and procedures available, and serves as a basis for orienting the norms of this pathology which affects women, and ever more frequently men.

\section{THE ROLE OF HPV IN THE CARCINOGENESIS OF GENITAL CANCER}

HPV infects the basal cells of the cervical epithelium and take advantage of the process of epithelial differentiation to synthesise proteins which allow them to assemble new viral particles. Infected epithelial cells activate their cellular defence mechanism in a revision of the DNA sequence before division. This process happens during a phase of the cellular cycle, and is lead by a protein cascade, among which $\mathrm{p} 53$ and $\mathrm{Rb}$ protein stand out. When the cell localises the viral DNA, in a perfectly controlled process, it tries to repair the error and, given that this DNA is excessively large to be eliminated, p53 and $\mathrm{Rb}$ lead the infected cell towards "programmed cellular death" by apoptosis, thus avoiding that this cell serves as an infectious propagator. HR-HPV strains are protected from this cellular mechanism by synthesising proteins which block the cellular defence system. It is considered that the viral transforming genes E6/E7 are responsible for the loss of control of cellular proliferation since the expression of high-risk oncogenic viral proteins is produced by the HPV E6 and E7 genes in neoplastic tissues, but not in healthy tissues. On the other hand, it has been observed that the HPV-16 E6 oncoprotein binds to $\mathrm{p} 53$, and that this binding causes the degradation of the latter, which is an important growth and cellular differentiation controller, partly due to stimulation of $\mathrm{p} 21$ and $\mathrm{p} 16$. Similar properties are observed for $\mathrm{pRb}$, besides the expression from E6 and E7 as an indispensable requirement for maintaining a malignant phenotype of the cancerous cells [8].

The process of blockage of $\mathrm{p} 53$ and Rb by the E6 and E7 proteins would not cause further problems if it did not result in cellular immortality. As a consequence of the blockage of the error repair system, the cell is not only incapable of eliminating the viral DNA, but it is also blocked from repairing intrinsic cellular DNA errors. At the same time, in associated genital cancers with HPV, a loss or reduction of the allelic expression of the class I molecules of the histocompatibility complex, which is closely involved in the recognition and presentation of surface antigens, is often found. Its alteration caused by HPV may explain why some cancers escape the immunological control provided by cells. HPV does not have specific cellular receptors, but a highly conserved surface molecule with vital cellular functions, which makes its use as a target for infection blockage impossible. As opposed to what happens with other virus, it does not appear that the surface receptors are involved in tissue and species specialisation or in tropism of the HPV [7].

It is known that in infections with high viral load, in which the immune system is unable to eliminate the infection, there is a higher risk of neoplastic transformation. Nonetheless, it has been proven that certain persistent infections with low viral load generate an effective tumoural phenotype: What, therefore, is the mechanism of immortality with low viral load? The proof that in the majority of carcinomas the viral DNA was fragmented and integrated into the cellular genome allowed this question to be answered. In the majority of cases, a section of viral DNA split in the E2 region, losing its capability to act on the regulatory area for viral genetic expression [9].

\section{ASSOCIATED CO-FACTORS FOR DEVELOPMENT OF ANAL-GENITAL CANCER}

It is well known that the vast majority of infected women spontaneously cure the infection, with only a small proportion suffering persistence - frequently sub-clinical which puts them at high risk of neoplastic development. In total, it is considered that $80-90 \%$ of infections cure themselves spontaneously, and that $10-20 \%$ persist. The mean duration of HPV infection is calculated to be 8-10 months. Curing the infection seems to give a certain level of protection against re-infections by the same viral genotype. Cases of persistent infection make up the high-risk group for neoplastic development [10].

Thus, despite being the necessary cause for cervical cancer, HPV is in no way a sufficient cause for the development of this tumour. Consequently, if only some infected women progress to suffer from HSIL/cancer lesions there are probably existing cofactors which, interacting with HPV, modulate the risk of development. 
Viral, genetic, sexual behaviour related and environmental associated factors have been described. Viral determinative factors for progression include: viral strain, viral load per cellular unit, phylogenetic variants and the integration with cellular DNA. Possible genetic cofactors include markers for genetic susceptibility, factors which regulate the cellular and humoral immune response to infection by HPV, HLA, and p53, among others. Among the environmental risk factors for infection by HPV in women are: age of the first coitus, hormonal contraceptive use, multiple sexual partners or partners with high-risk behaviour, high parity, poor hygiene and smoking [11].

A study carried out in Barcelona demonstrated the low prevalence of HPV in monogamous couples [12].

With respect to males we observe the majority of associated factors described earlier for women. Moreover, we should add other environmental factors such as poor hygiene, inadequately treated phimosis combined with the retention of sperm. The abuse of PUVA, balanitis and chronic irritable states. To this we should add the performance of circumscription or not in the neonatal period [13].

Other cofactors are the existence of other sexually transmitted diseases (STD), such as hepatitis B, C, Chlamydia trachomatis, herpes virus, syphilis, sexual relations with anal penetration and HIV, since, a group with higher risk of invasive carcinoma progression are HIV positive patients, especially if they do not respond to or adhere with antiretroviral therapy, promoting an immunosuppressive state [14-16].

Another special situation is during pregnancy. Cytology is performed on all pregnant women who were not checked the previous year during the first check-up after conception. Squamous intraepithelial lesion (SIL) cytology requires a diagnostic study with a colposcope similar to that performed on non-pregnant women. The result of detection during pregnancy of HPV DNA in the cervix or vagina should not modify the performing of developmental follow-up or the proposal for the delivery plan. The risk of vertical transmission or persistent infection in the newborn is very low. Directed biopsies are not routinely performed, but they are when it is necessary to rule out invasion. They may be carried out at any time during gestation, adopting the due precautions for correct haemostasis [17-19].

\section{A DIAGNOSTIC APPROACH OF THE SCREENING SITUATION IN SPAIN AND EUROPE}

Screening programmes in Spain are fulfilled unequally and have poor coverage. The target population are heterogeneous and are identified according to unclear criteria. The cytology laboratories frequently use their own terminology when they issue reports. It is difficult to know the screening cost of cervical cancer in Spain, as no standardised procedure exists for cost analysis. Not all the population censuses of tumours record the carcinomas in situ of the cervix. The average screening coverage in Spain is $75 \%$ with great differences between one community and another. Around $25 \%$ of Spanish women are kept away from preventive follow-up, this group is comprised of women older than 55 years old, of low social level and living in rural areas. Therefore, new proposals for modification of the screening programme in Spain exist based on the recommendations of the 2006 Spanish Consensus for Secondary Prevention of the Cervix and on resolution 287 of the European Council, which transcribes the conclusions of the meeting held on $10^{\text {th }}$ June 2008 in Luxembourg, related to the volume of cancers in the European Union and mechanisms to reduce it. According to this, screening should be mixed, with active calling of the women whom do not attend the health centres within the time established. Thus, screening should commence at 25 years old or within the first three years after starting sexual relationships. Women without sexual relationships are excluded from the programme. A cytology should be performed, double sample exo/endocervix every three years until 30 years of age, the age at which a test for HPV DNA by PCR should be performed. Those patients with negative test results would be revised every 5 years. The screening finishes at 70 years old when correct compliance of the screening program has been followed. The Bethesda (Table 1) system should be accepted, without exception, to inform about the results. Diagnosis of HG-CIN should be incorporated into the population tumour records. Cost-benefit analysis programmes should be started for the screening programmes implemented [20, 21].

\section{FORMS OF HPV INFECTION OBSERVED}

The spectrum of epithelial anomalies, considered as precursors or pre-colonizers which are considered as progressive, have been named in various ways. Thus, the concept and terminology of pre-malignant alterations of the cervical epithelium have developed in parallel with the advance in the knowledge of its biology and cervical intraepithelial development (CIN), which unites them classifying the lesions in three grades (Figs. 1, 2). This terminology is still used at present in histological diagnosis. In 1988, the Bethesda system was proposed for describing cytological alterations, which includes new concepts on infection by HPV. In this meeting, the quality review of the samples was one of the most innovative suggestions. After various years of trials, the system was evaluated in a second workshop in 1991. In 2001, this classification was reviewed again and slightly modified and the term neoplasia was substituted for the term squamous intraepithelial lesion (SIL), with two grades: low grade (LSIL) and high grade (HSIL). This division into two groups is justified by the evidence that LSIL basically corresponds to viral infections, which are generally self-limiting, and only exceptionally develop into carcinoma; whilst HSIL corresponds to true pre-malignant changes [22, 23] (Table 1).

The determination of HPV via PCR in intraepithelial lesions has shown that in LSIL highly heterogeneous strains are identified, with high and low oncogenic risk, while in the majority of HSIL high risk strains are found. For anatomicalpathological diagnosis, the sample is obtained by colposcopic examination. It is necessary to apply any treatment a posteriori.

At present, after performing a satisfactory colposcopy, cases of histologically confirmed CIN I, are periodically controlled by cytology and colposcopy every six months for two years. The case should be individually reviewed according to the age and characteristics of the patient [24]. 
Table 1. The 2001 Bethesda System for Reporting Cervical Cytological Diagnosis

\section{Specimen adequacy}

Satisfactory for evaluation

Presence or absence of endocervical or transformation zone components or other quality indicators such as partially obscuring blood or inflammation

Unsatisfactory for evaluation (specify reason)

Specimen rejected or not processed (specify reason)

Specimen processed and examined, but unsatisfactory for evaluation of epithelial abnormalities (specify reason)

General categorization (optional)

Negative for intraepithelial lesion or malignancy

Epithelial cell abnormality

Other

Interpretation/result

Negative for intraepithelial lesion or malignancy

Organisms

Trichomonas vaginalis

Fungal organisms morphologically consistent with Candida species

Shift in flora suggestive of bacterial vaginosis

Bacteria morphologically consistent with Actinomyces species

Cellular changes consistent with herpes simplex virus

Other non-neoplastic findings (optional to report)

Reactive cellular changes associated with:

Inflammation (includes typical repair)

Radiation

Intrauterine contraceptive device

Glandular cells status posthysterectomy

Atrophy

Epithelial cell abnormalities

Squamous cell

Atypical squamous cells (ASC)

ASC of undetermined significance (ASC-US)

ASC, cannot exclude high-grade squamous intraepithelial lesion (ASC-H)

Low-grade squamous intraepithelial lesion (LSIL)

Encompassing: human papillomavirus, mild dysplasia, and cervical intraepithelial neoplasia (CIN) 1

High-grade squamous intraepithelial lesion (HSIL)

Encompassing: moderate and severe dysplasia, carcinoma in situ, CIN 2, and CIN 3

Squamous cell carcinoma

Glandular cell

Atypical glandular cells (AGC)

Specify endocervical, endometrial, or glandular cells not otherwise specified

Atypical glandular cells, favor neoplastic

Specify endocervical or not otherwise specified

Endocervical adenocarcinoma in situ (AIS)

Adenocarcinoma

Other (list not comprehensive)

Endometrial cells in a women 40 years or older

Automated review and ancillary testing (include if appropriate)

Educational notes and suggestions (optional) 


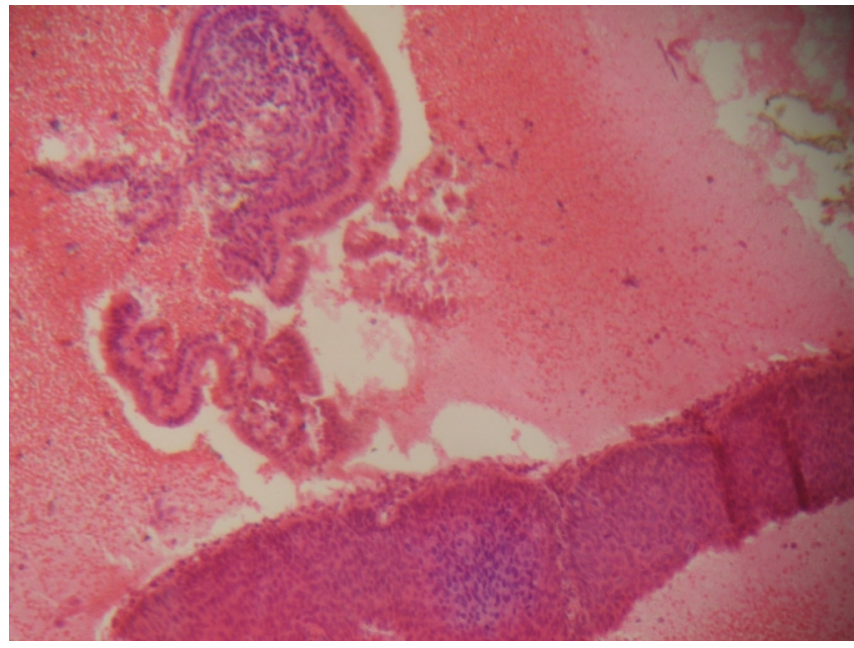

Fig. (1). Exocervix without alterations.

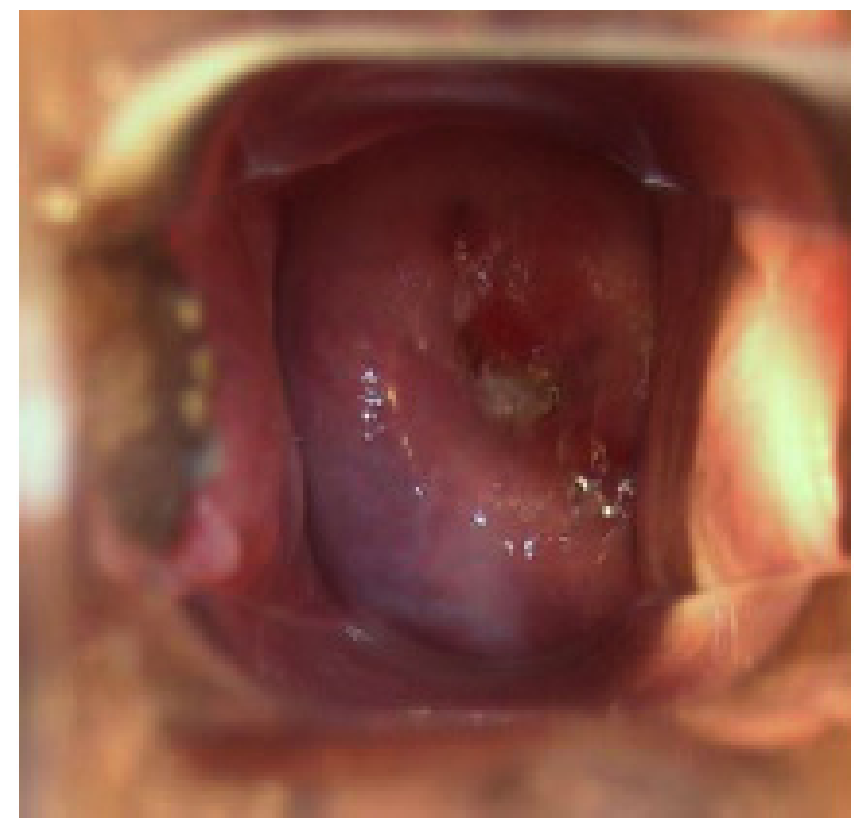

Fig. (2). Whitened macular papules in the cervix, characteristic after the application of acetic acid.

In the case of CIN 2 or CIN 3, destructive methods, fundamentally cryocoagulation, since other methods are infrequently used nowadays, give advantages such as the ease of application in surgery, and the fact that they are painless and economical; nonetheless we cannot perform histological confirmation or evaluate the safety margins and depth of the lesions. Laser vaporisation is effective, adds safety, although it is expensive and does not allow for anatomical-pathological confirmation. With respect to excision methods, cold scalpel conisation is practically never performed except in the case of in situ adenocarcinoma. The most commonly used techniques at present are conisation with a diathermic lance or laser. The results and percentages of relapse or persistence (2.5-3\%) are similar for both techniques. With respect to hysterectomy it would not be indicated as a primary treatment, except in exceptional cases: associated organic pathology which justifies its use, in patients who have completed their families or when a cone is obtained with affected borders in patients that do not want further gestations, or in cases of impossible or difficult follow-up. Follow-up after conisation should be performed periodically each 6 months via cytology and colposcopy and it is advisable to carry out a post-therapeutic DNA test for HPV, since its presence or not is used as a prognostic criterion for the disease. In the case of negative DNA, the percentage of relapses is $1.6 \%$, as opposed to $49 \%$ in the case of positive. With respect to the possible risks of these techniques for the development of posterior gestations, cold scalpel conisation carries with it a higher risk of preterm delivery. In a recent publication on the effects of lance conisation it was observed that, even after correcting other associated variables, the risk of preterm delivery for these patients was $14.5 \%$ against $5 \%$ in the control group. Preinvasive lesions of the cervix affect a great percentage of patients and are much higher than in carriers of infiltrative carcinoma. The therapeutic options, although conservative and limited to high grade lesions (CIN2/3), are not innocuous or exempt from sequels, although they are not serious, but rather the recovery rates are high and relapses low, and definitely susceptible to a second or third therapeutic option. Periodical post-treatment check-ups are recommended $[25,26]$.

A special situation with respect to treatment is pregnancy. Conisation is not advisable during this period due to the associated haemorrhagic morbility. In the absence of invasive cancer, vaginal delivery should be recommended, with later colposcopic control. Treatment of the CIN should be postponed until after the delivery. Other investigations supply figures that $32 \%$ of men with low grade intraepithelial penile neoplasia were sexual partners of women with CIN $2 / 3$, whilst $72 \%$ of men with high grade penile intraepithelial neoplasia had CIN 2/3 [27].

In situ adenocarcinoma (ISA) of the endocervix originates in the reserve cells located below the cylindrical epithelium. More than $60 \%$ are associated to a squamous lesion of the cervix, from a simple condyloma, to a more advanced lesion, even an infiltrative epidermic carcinoma. They represent 5 to $8 \%$ of all cervical carcinomas. ISA is a new category which corresponds to a series of specific morphological alterations. It is a lesion which is frequently difficult to diagnose, since it is located in the endocervical area of the endo-exocervical union, even sometimes at the base of a gland whose superficial epithelium has been substituted by a squamous metaplasia and, therefore, the cells are not represented in the sample. This is also as the cells are not, for various reasons, recognised as atypical, or since they are confined to an endocervical polyp. In recent decades an increase in ISA has been observed, as well as in invasive adenocarcinoma and adeno-squamous carcinoma. The presence of HPV has been observed in $90 \%$ of these lesions. The association of ISA/HPV is very strong, with an increase in risk of 68.7 times (95\% CI, 36.2 to 130.5). Unlike squamous cancers, in which HPV-16 is the most frequent, in glandular cancers HPV-18 are the most prevalent or other phylogenetically related strains (HPV 39, 45 and 59). Although the reasons are unknown for this specificity, glandular carcinomas share the same risk factors as squamous carcinomas. ISA is located in the transformation area and is associated in a third of the cases to SIL, generally HSIL. Very frequently, it is the diagnostic study of cytology with abnormal squamous cells which allows us to reach the diagnosis of adenocarcinoma in situ. Histologically, the 
transition is brusque between adenocarcinoma and normal epithelium. The diagnosis is based on architectural and nuclear criteria. In women with atypical glandular cell cytology (ATC), the risk of a high grade squamous or glandular lesion is over 30\% [28].

The age at which we find development of invasive carcinoma is placed between 45 and 50 years old. Its anatomical-pathological forms are multiple, but we may basically differentiate the epidermal carcinomas which colonise the stratified epithelial wall which covers the cervix and the adenocarcinoma which colonises the glandular epithelium of the endocervix. This tumour, if it is untreated, propagates through the lymphatic system from the ganglions of the internal and external iliac chain to the pre-aortic level and the upper levels and to the neighbouring tissues and organs: parametrium, vagina, bladder and rectum in the more advanced stages.

In early stages, the tumour may be a chance finding on carrying out a cytological diagnosis. Nonetheless, frequent symptoms are: irregular metrorrhagia not associated to menstruation, and coitorrhagia; localized pain in the hypogastrium or radiating to the lower limb or lumbar area in more advanced stages; and oedema of the lower limbs.

The diagnosis is clinical or colposcopic and, of course, should be confirmed with anatomical-pathological study. Once this is established we should proceed to an extended study protocol to establish the stage of the process and decide which therapeutic option would be most suitable. The development classification used is the stage classification from the International Federation of Gynaecology and Obstetrics (FIGO) based on the dissemination form of the tumour. Four stages are differentiated.

Stage I: Cervical carcinoma confined to the cervix

Stage II: Tumour invades beyond the uterus but not to the pelvic wall or to the lower third of the vagina.

Stage III: Tumour extends to the pelvic wall and/or involves the lower third of the vagina and/or causes hydronephrosis or non-functioning kidney.

Stage IV: Tumour invades the mucosa of bladder and/or rectum and/or distant metastasis.

Treatment is fundamentally based on the tumoural stage, age of the patient and the presence or not of associated pathology. Conisation is acceptable management if the depth of the invasion is found to be less than $3 \mathrm{~mm}$ from the basement membrane. The most frequent thing is to perform radical surgery associated with, on occasions, radiotherapy and chemotherapy, before and after surgery. In stages II-III the most commonly used option is chemotherapy combined with radiotherapy. In some cases of vesical or digestive affection, more complex radical surgery may be chosen. Survival reduces on increasing the stage and varies from $90 \%$ for stage I to figures below $10 \%$ for more advanced stages [24, 29, 30].

\section{VULVAR INTRAEPITHELIAL NEOPLASIA}

The International Society for the Study of Vulvar Diseases (ISSVD) was the first to establish a classification of vulvar diseases. In 1986 [31], this society established the classification of intraepithelial neoplasias of the vulva and introduced the term Vulvar Intraepithelial Neoplasia (VIN) as a pre-invasive lesion differentiated from the squamous variety with slight, moderate or severe atypia/carcinoma in situ, which corresponds to VIN 1, 2 and 3. The nonsquamous form includes Paget disease and in situ melanoma. Later a new classification was proposed, giving rise to the terms Low Grade VIN (LG-VIN) (Fig. 1) and High Grade VIN (HG-VIN) (Figs. 3, 4), which encompassed the anterior terms (Table 2) [31, 32].

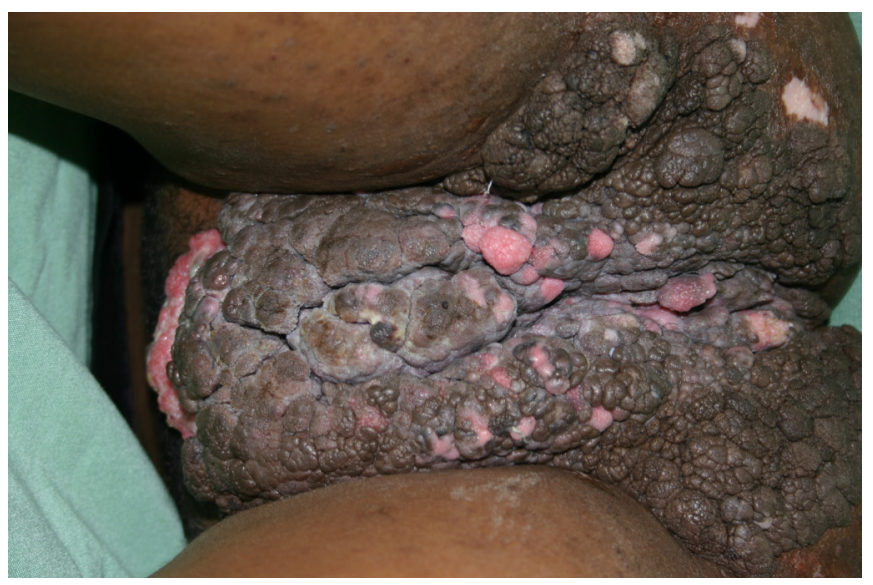

Fig. (3). Giant condylomas which obscure anatomical orifices (low grade VIN).

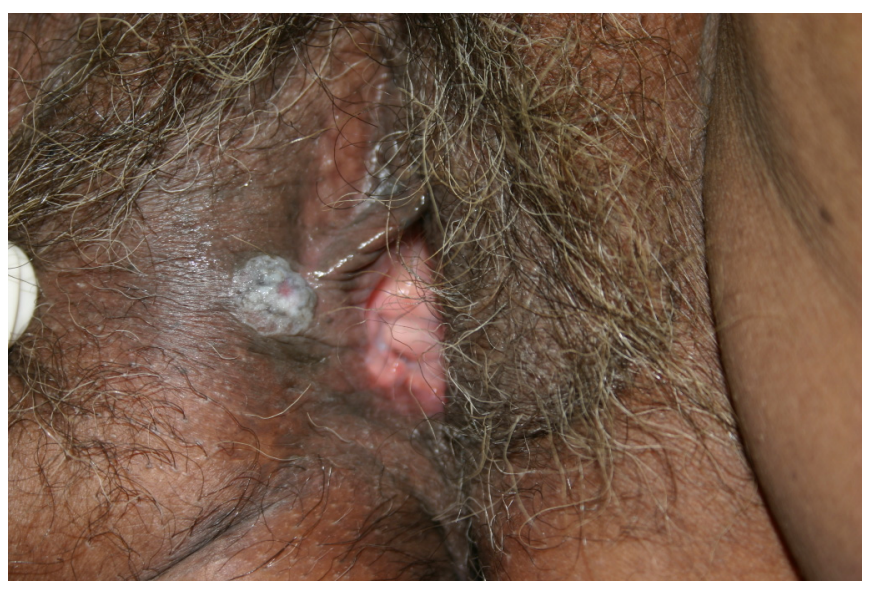

Fig. (4). High grade VIN of a post-menopausal woman.

Table 2. Proposed 2003 Terminology of Squamous VIN (ISSVD Newsletter, January 2004) *

\begin{tabular}{|ll|}
\hline I. & Low grade VIN (LG-VIN) \\
II. & High grade (HG-VIN) \\
& a. HG-VIN-AG, usual type \\
& $-\quad$ HG-VIN, warty type \\
& b. HG-VIN, differenciated type \\
& c. HG-VIN, unclassified type (NOS), may include rare \\
& types, i.e. HG-VIN pagetoid type. \\
*Society for the Study of Vulvar Disease.
\end{tabular}

The basaloid and condylomatous histological types are generally related to HPV, generally appear in young women from 20 to 40 years old, and their prognosis is favourable. The differential type which generally appears in older 
persons has a controversial relationship to HPV and has a poor prognosis (Table 2).

In the later two decades there has been an important reduction in mortality due to vulvar cancer, whilst its incidence has remained stable. On the contrary, the incidence of VIN has doubled, especially in young women [7]. Among the causes which may explain these phenomena are early diagnosis and a real increase in the incidence of VIN, due to changes in sexual behaviour, with a greater exposition to HPV infection, which supports the viral theory for VIN. Thus, its justification lies in the fact that these organs have the same embryonic origin and share the same oncogenic stimuli. In a bibliographical review, $32.8 \%$ of patients with VIN 3 had synchronous or metachronic neoplasias in other areas of the LGT. In patients with VIN 3, more than $38 \%$ had associated CIN. It is difficult to know the development of VIN and its progression to carcinoma, due to the low number of patients and due to the fact that the majority have been treated by one form or other. In recompilations of records until the 90 's, from a total of 35 untreated patients with VIN 3, six developed into carcinoma (17\%). VIN 3 with bowenoid characteristics quickly develops into invasive carcinoma (Fig. 5). The early diagnosis and treatment of VIN avoids the risk of invasive cancer. Even so, progression occurs in $4-7 \%$ of patients [7].

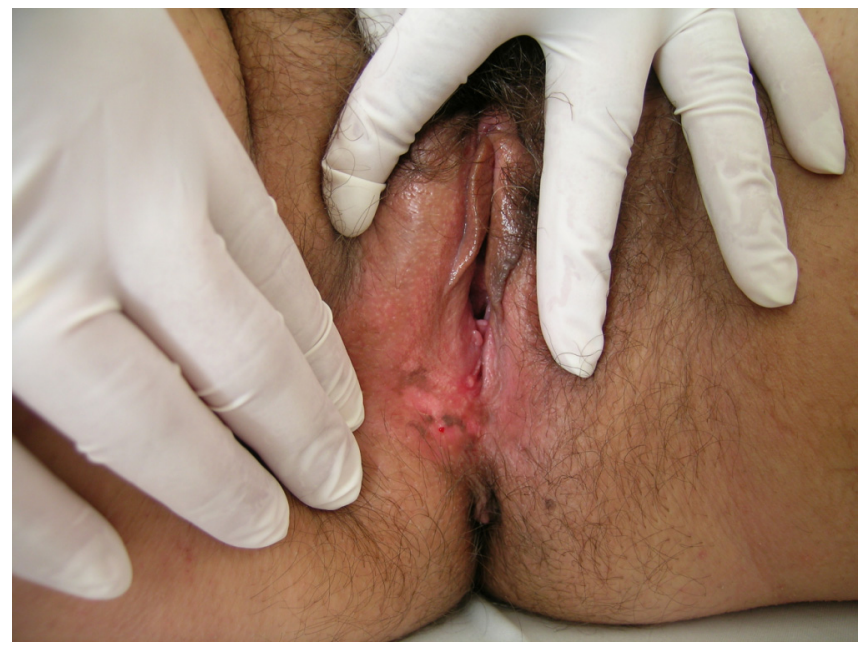

Fig. (5). High grade VIN in a smoker patient

In the therapeutic area, it is necessary to mention, firstly, the modifiers of the immune response, among which imiquimod stands out, since it has introduced changes in the approach to certain pathologies. It gives excellent aesthetic results and administration by the patient themselves is well accepted. Other treatments for VIN such as interferon are used in combination with isotretinoin. Nevertheless, histological methods persist and regression is scarce. Cidofovir improves results of surgical excision of condylomas in HIV positive patients, and is found to be effective in some cases of VIN. Topical chemotherapy with 5-fluoracil has been abandoned [7].

The treatments of choice, after discounting expectant behaviour and/or medical treatment are: 1) Wide local extirpation; 2) partial or total cutaneous vulvectomy, followed by cutaneous graft; 3) suppression of the lesion via cryotherapy or laser; and 4) the use of combined techniques of excision and ablation. In any case, we should be prudent in the treatment, since it generally appears in young women and extensive and mutilating surgery causes great sexual and psychological problems, which explains the importance of diagnosis and early treatment. In young pregnant women it is advisable to watch and wait until after delivery. If the lesion is unifocal an excision may be performed. On the other hand, if it is multifocal, it is advisable to perform a partial vulvectomy or combined treatments and the use of combined treatments of excision and laser is advisable. If the lesions are very extensive, a total vulvectomy is recommended. In these we should extirpate the skin to the subcutaneous cellular tissue. The clitoris should always be conserved. Follow-up after treatment should be performed every 4 or 6 months via exhaustive clinical-vulvoscopic exploration and biopsy in suspicious lesions. Follow-up should be, at least, for 2 years, since recurrences are frequent. Immunodepressed patients and old-age patients should follow a strict control as they have a higher risk of developing an invasive cancer [7, 32].

The structural diversity of the tissues of the vulva explains the great variety of malignant proliferations which may develop there. The vulva is the fourth most common location for development of cancer of the female genital apparatus. Nonetheless, invasive cancers of the vulva only constitute 3 to $5 \%$ of all cancers of the female genitalia. They are the next most frequent types after uterine and endometric cancers. Traditionally the mean age has been placed between $70-85$ years old, with the condition being very rare in young patients. At present the epidemiological pattern has varied with the apparition of lesions in people below 45 years old. Among the aetiological factors immunosuppressive drug use, chronic granulomatous disease, scleroatrophic lichen, hyperplasia of the squamous cells and VIN are highlighted. High grade VIN would be the precursory lesion, which appears 10 years before. Keratinising carcinoma is associated with scleroatrophic lichen and hyperplasia of squamous cells. Its prognosis is unfavourable [33].

Squamous carcinoma (SC) is the most frequent histological type in the statistics, as it represents $90 \%$ of all primary neoplasias. We shall only study this type in this paper. In second place for frequency are the melanomas, followed by verrucoid carcinoma, base cell carcinomas, Paget disease and, finally, sarcomas [34] (Figs. 6, 7).

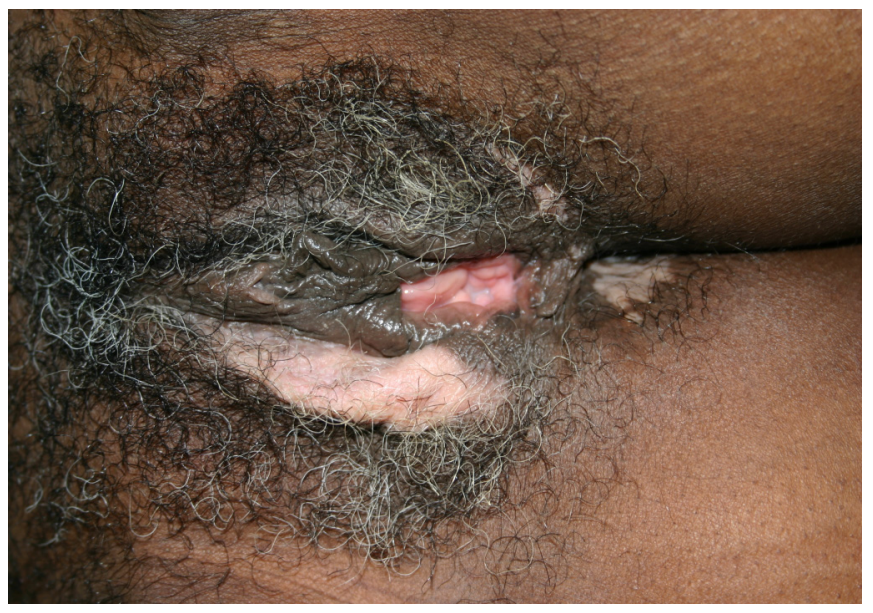

Fig. (6). Squamous cell carcinoma in a HPV positive patient. 


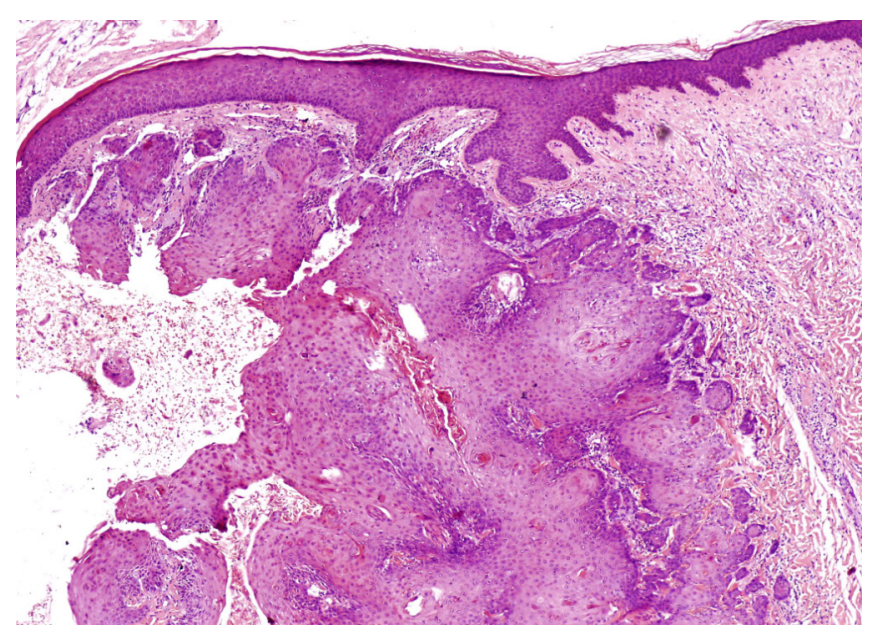

Fig. (7). Well differentiated invasive squamous cell carcinoma shoving multiples areas of keratinization (H\&E x100).

In typical cases of $\mathrm{SC}$, it is a disease of post-menopausal women, with an average age of 65 years old, although the majority of the series published are of ages between 30 and 90 years old. Patients younger than 50 years old at the time of carrying out the first diagnosis correspond to $10 \%$ of the cases. From the keratinising forms, $70-75 \%$ are well differentiated, $20 \%$ are fairly well differentiated and $10 \%$ show little differentiation [34].

The most common symptoms associated with vulvar SC include: long-term pruritus, flux or exudate which occasionally has a bad smell and non-menstrual bleeding, pain and dysuria. In its early stages, vulvar cancer is seen as a hardened lump, occasionally hyperkeratinous, of variable colour ranging from white to slightly erythematous. During this phase it is easy for the lesions to appear masked by the association with VIN, sclerotic lichen, hyperplasia of the squamous cells, genital atrophy or even over-infection with lichenification, due to excessive scratching by the patient. In more advanced studies, the morphology is generally more evident and is seen as ulceration accompanied by reddening, polypoid or nodular or, even with a whitish hyperkeratinous colour and may accompany a palpable inguinal lump (Figs. $\mathbf{5 , 6 )}$. In $10 \%$ of cases the lesions are multicentric.

In order to classify vulvar carcinoma into stages according to the classification of the FIGO Oncological Committee [35] (Tables 3, 4), in stage IA a simple excision of the lesion is performed and, generally, ganglionary inguinal dissection is not required. This justifies the realisation of the sentinel ganglion in case of great doubt [36].

In the case of stage IB, if the lesions are lateral, a local radical excision or radical hemivulvectomy with homolateral lymphadenectomy may be performed. If the lesions are central, the vulvectomy should be radical and the lymphadenectomy bilateral, and it is advisable to use triple incision. In stage III, the treatment choice is to perform radical vulvectomy, which may be more or less wide, according to the extension of the inguinal and femoral lymphadenectomy, and complementary radiotherapy. Radical radiotherapy may be an option in patients where aggressive surgical treatments are contraindicated. They have good levels of long-term survival $[35,36]$.

The complementary technique of performing surgery of the sentinel ganglion is indicated in:

- $\quad$ Certain cases for stage IA.

- $\quad$ SC in stage IB or II with a tumour of less than $3 \mathrm{~cm}$.

- Depth of invasion greater than $1 \mathrm{~mm}$.

- Clinically negative lymphatic inguinal-femoral ganglions.

Chemotherapy and radiotherapy are used in advanced studies as a neoadjuvant for standard surgical treatment [3436].

Table 3. Carcinoma of the Vulva Staging

\begin{tabular}{|c|c|c|}
\hline FIGO/Stage & & TNM/Categorías \\
\hline & Primary tumour cannot be assessed. & $\mathrm{TX}$ \\
\hline 0 & Carcinoma in situ. & Tis \\
\hline I & Tumour confined to vulva or vulva and perineum, $2 \mathrm{~cm}$ or less in greatest dimension. & $\mathrm{T} 1$ \\
\hline IB & Tumour confined to vulva or vulva and perineum $\leq 2 \mathrm{~cm}$ in greatest dimension and with stromal invasion $1.0 \mathrm{~mm} *$. & $\mathrm{T} 2$ \\
\hline II & $2 \mathrm{~cm}$ Tumour confined to the vulva or vulva and perineum, $>2 \mathrm{~cm}$ in greatest dimension. & $\mathrm{T} 3$ \\
\hline III & Tumour invades any of the following: lower urethra, vagina, anus & $\mathrm{T} 4$ \\
\hline \multicolumn{3}{|l|}{ IV } \\
\hline
\end{tabular}


Table 4. Carcinoma of the Vulva/Stage Grouping

\begin{tabular}{|c|c|c|c|}
\hline \multirow{2}{*}{ FIGO/Stage } & \multicolumn{3}{|c|}{ UICC } \\
\cline { 2 - 4 } & T & N & M \\
\hline \hline 0 & Tis & N0 & M0 \\
\hline IA & T1a & N0 & M0 \\
\hline IB & T1B & N0 & M0 \\
\hline II & T2 & N0 & M0 \\
\hline III & T1 & N1 & M0 \\
\hline & T2 & N1 & M0 \\
\hline & T3 & N1 & M0 \\
\hline IVA & T1 & N2 & M0 \\
\hline & T2 & N2 & M0 \\
\hline & T3 & N2 & M0 \\
\hline IVB & T4 Any T & Any N & M0 \\
\hline
\end{tabular}

Modified from FIGO: International Federation of de Gynaecology \& Obstetrics, Committee on Gynaecology Oncology, 2000.

UICC: Union Internationale Contre le Cancer.

Regional lymph nodes $(\mathrm{N})$.

NX - Regional lymph nodes cannot be assessed; NO - No regional lymph node mestastasis; N1 - Unilalateral regional lymph node metastasis.

N2 - Bilateral regional lymph node metastasis.

Distant metastasis $(\mathrm{M})$.

MX - Distant metastasis cannot be assessed; M0 - No distant metastasis; and M1 Distant metastasis (including pelvic lymph node metastasis).

Histopathologic grades (G).

GX - Grade cannot be assessed; G1 - Well-differentiated; G2 - Moderately differentiated; G3 - Poorly or undifferentiated.

Relapses are generally due to insufficient resection. Nonetheless, later malignisation may occur in a neighbouring area. Its treatment should be individualised, but, as a general norm, in cases of local relapse a wide excision is recommended. If the relapses are pelvic or in distant organs radiotherapy and chemotherapy should be evaluated [34].

Survival depends, to a great extent, on the pathological state in which the inguinal ganglions are found. In patients with operable disease with ganglionary complication, the general survival rate is $90 \%$. Nonetheless, when there is ganglionary complication, the general survival rate at 5 years is $50-60 \%$. The risk factors responsible for ganglionary metastasis are the clinical ganglionary state, age, level of differentiation, tumoural state, tumour thickness, stromatic depth and the presence of invasion of the capillary lymphatic space. In general, approximately $30 \%$ of patients with operable disease show ganglionary dissemination (Tables $\mathbf{3}$, 4) $[36]$.

\section{Anal Intraepithelial Neoplasia (AIN)}

The cervix and anal canal share the same embryonic structure. Both derive from the cloacal membrane and are areas of endodermal and ectodermal fusion, that is, they form a squamous-columnar joint which is generally subject to metaplastic phenomenon and may develop dysplasia when HPV is present. Some comparisons based on tumoural records have estimated that the incidence of anal canal cancer in homosexual males is similar to the incidence of cervical cancer in populations without screening programmes, being especially relevant in homosexual males infected with HIV. Histories of perianal condylomas are observed in $50 \%$ of MSM and $30 \%$ of women with AIN. It is estimated that HPV is present in $90 \%$ of anal cancers. Occasionally AIN is asymptomatic. Nevertheless, pain, tenesmus, pruritus and/or haemorrhage may occur. Diagnosis is based on cytology, digital exploration and anuscopy. There is no data for recommending systematic screening. Treatment consists of tissue resection which reaches the free edge. With respect to the infiltrative form, the most common symptom is haemorrhage. Treatment consists of surgery and radiotherapy associated with chemotherapy. Survival for infiltrative cancer is around $40-70 \%$ at 5 years. At present, regimes with associated chemotherapy have achieved survival between $72-89 \%[37,38]$.

PIN, as with vulvar intraepithelial neoplasia (VIN), covers all precancerous lesions of the squamous epithelium of the penis. It is characterised by the presence of cellular atypia. Its diagnosis and early treatment avoids development into cancer of the penis.

Although the prevalence is unknown, over recent years we have observed an increase in this pathology. This is due, in part, to the increase in infection by HPV, on the one hand, and the use of new diagnostic techniques, on the other. PIN was previously defined as a pre-invasive lesion which is differentiated from the squamous variant with slight, moderate or severe atypia/carcinoma in situ. This includes Bowen disease, Queyrat erythroplasia and bowenoid papulosis, and corresponds to PIN I, II and III. Later, as in the classification of vulvar diseases and, especially, for classifying VIN, a new classification was accepted, giving rise to the terms Low Grade PIN (LG-PIN) and High Grade PIN (HG-PIN) (Fig. 8), which cover the earlier terms [13, 39]. As with VIN so with PIN we have seen a considerable increase in cases in young men [40,41].

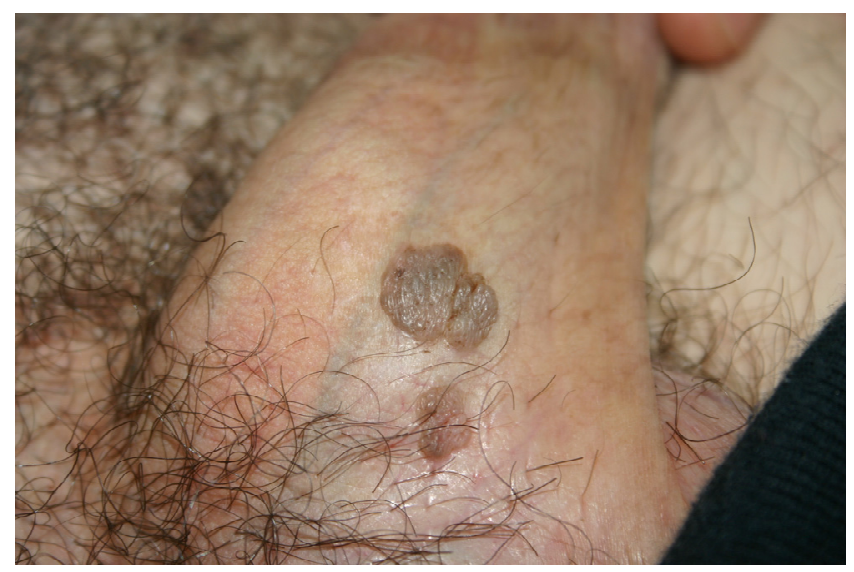

Fig. (8). Pigmented condylomata acuminata with foci of high grade PIN.

PIN is frequently asymptomatic. Nonetheless, the most common symptom is pruritus, which is generally also the first symptom. The morphology and appearance are variable. In young patients they can have a brown, red, violet and grey-white appearance. The lesions may be macular and/or papular. Unlike VIN it is not generally polychromatic, and does not show mixed morphology. Blood vessels are readily 
visible within the lesions. The lesions are multiple, with multifocal growth and a tendency to grow together. In older patients, the plaques are generally velvet red or white and with a smooth or rough surface; at the same time the lesions grow centrifugally, covering extensive areas [13].

Histopathologically, PIN is characterised by a loss of maturity of the epithelial cells, associated with hyperchromasia, nuclear pleomorphism, abnormal mitosis and cellular stacking. They may have dyskeratotic cells, "round bodies", hyperkeratinosis, parakeratinosis and pigmenti incontinence. The grades of PIN are classified according to the affectation of the epithelium. PIN includes Bowen disease, Queirat erythroplasia and a papular variant of PIN 3 (which includes bowenoid papulosis), and it is responsible for the basaloid forms and condylomas of the carcinomas of the penis. Other histological variants of PIN are differentiated PIN and unclassified PIN.

The origin of the keratinizing forms of carcinoma of the penis are under discussion. Scleroatrophic lichen are found associated with well-differentiated epidermal carcinomas and verrucous carcinoma, but in association with HPV 16. Hyperplasia of squamous cells is the most common form of non-HPV precursory lesion associated with invasive carcinoma (Fig. 9) [13].

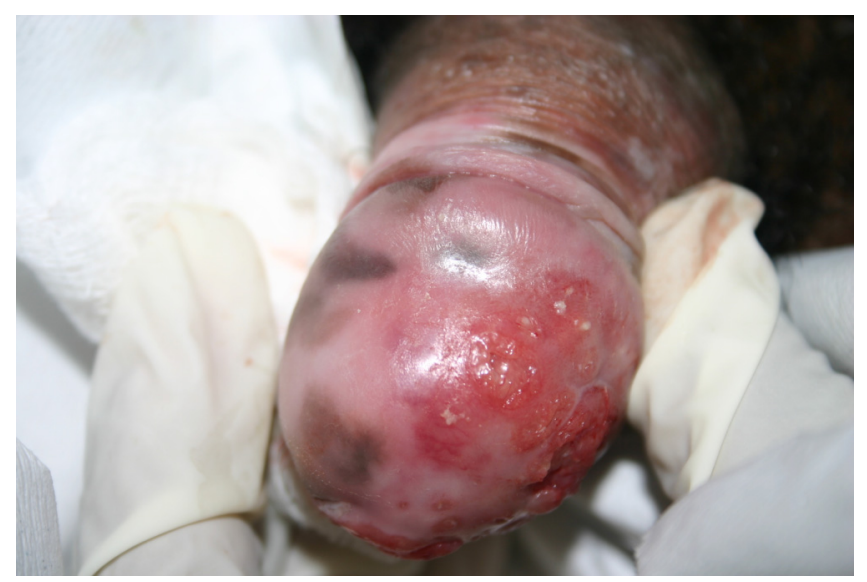

Fig. (9). PIN which develops into invasive carcinoma.

The diagnosis should include analysis through the realisation of peneoscopy in which the most noteworthy findings should be recorded of morphology of the lesions, extension etcetera. The acetic acid test reveals acetowhite epithelium which is highly useful for the identification of sub-clinical lesions, above all in the hands of trained personnel.

The differential diagnosis of PIN in young patients should be done, first, with scleroatrophic lichen and in the case of pigmented lesions, with the nevoid lentigines, seborrhoeic keratinosis and even malignant melanoma, among others. The erythematous lesions should be differentiated from the plane lichen, psoriasis, extramammary Paget disease and balanitis in general. In older patients we should distinguish the whitish lesions, which should be differentiated from the spiked condylomas and scleroatrophic lichen. The erythematous lesions should be differentiated from simple lichen, psoriasis and extramammary Paget disease [13, 42].
The treatment is occasionally conservative. The classical topical treatments fell into disuse in favour of imiquimod. At present, with the arrival of the first prophylactic vaccines, in especial the tetravalent HPV vaccine, new hope has arisen for the elimination of precursory lesions and their later development to invasive carcinoma in those cases associated with HPV. If the affectation is in the prepuce a postectomy is carried out. If the affectation is in the gland, we may use laser therapy, micrographic Mohs surgery or directly perform a glandectomy. We may also fall back on the use of combined techniques [13, 43, 44].

Within malignant penile tumours, the most frequent type is squamous carcinoma, which develops from the mucous membrane of the preputio and more quickly in the skin of the body of the penis (Fig. 10). It is observed in men over 50 -years old, with a peak in incidence at 60-69 years old. It represents $2 \%$ to $5 \%$ of male urogenital cancers, although it has a highly variable incidence, which in some countries reaches $10 \%$ of male malign pathologies, which is in direct relation with cultural and hygienic habits. The reduction in incidence in developed countries makes it difficult to carry out multi-centre studies, thus correct staging of the disease is one of the fundamental passes for therapeutic treatment.

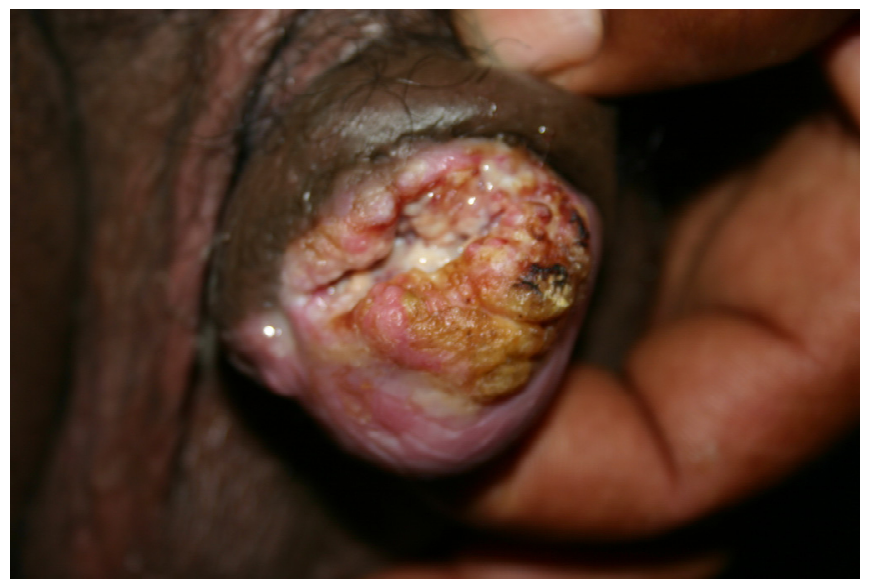

Fig. (10). Invasive carcinoma: Extensive exophytic mass, ulcerous, which invades the gland and projects beyond the preputial-balanic fold.

Clinical presentation is variable. It may be seen as a hardened area, a nodule, a small or large-sized exophytic verrucous lesion.

It is frequently ulcerate and oozing. Its visualisation is more difficult if the patient is suffering from phimosis. It is often accompanied by a foul-smelling urethral exudate. Despite being a highly visible organ, the patient generally first comes to the doctor's surgery in late stages of disease. Normally the first sign is the appearance of inguinal adenopathy. It is important to highlight that, although the first diagnosis may be visual, at the time of consultancy the definitive diagnosis will be given by biopsy of the lesion.

No universal system of staging exists. No classification exists which adequately correlates the clinical situation with the therapeutic option and prognosis. The Jackson classification (Table 5) has been used, but this is centred on exclusively clinical parameters, and neither includes histopathological parameters of tumoural invasion, nor unior bilateral criteria of affected ganglia, aspects which are 
highly important to evaluate the prognosis of the disease. In 1987 the Unión Internacional contre la Cáncer (UICC International Union for Cancer Research) and the American Joint Committee on Cancer (AJCC) agreed on the various stagings of TNM for all tumours. This classification and the earlier variations are shown in Table 2, with the most recent revision being in 1997. The most frequently used classification is the one based on TNM (Table 6) [13, 42].

\section{Table 5. Jackson's Classification (1966)}

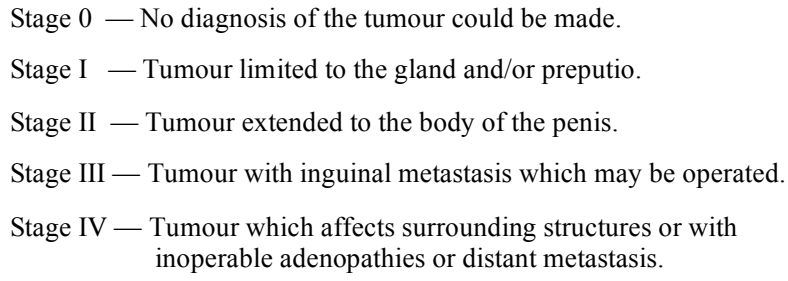

Table 6. Carcinoma of the Penis/Stage Grouping

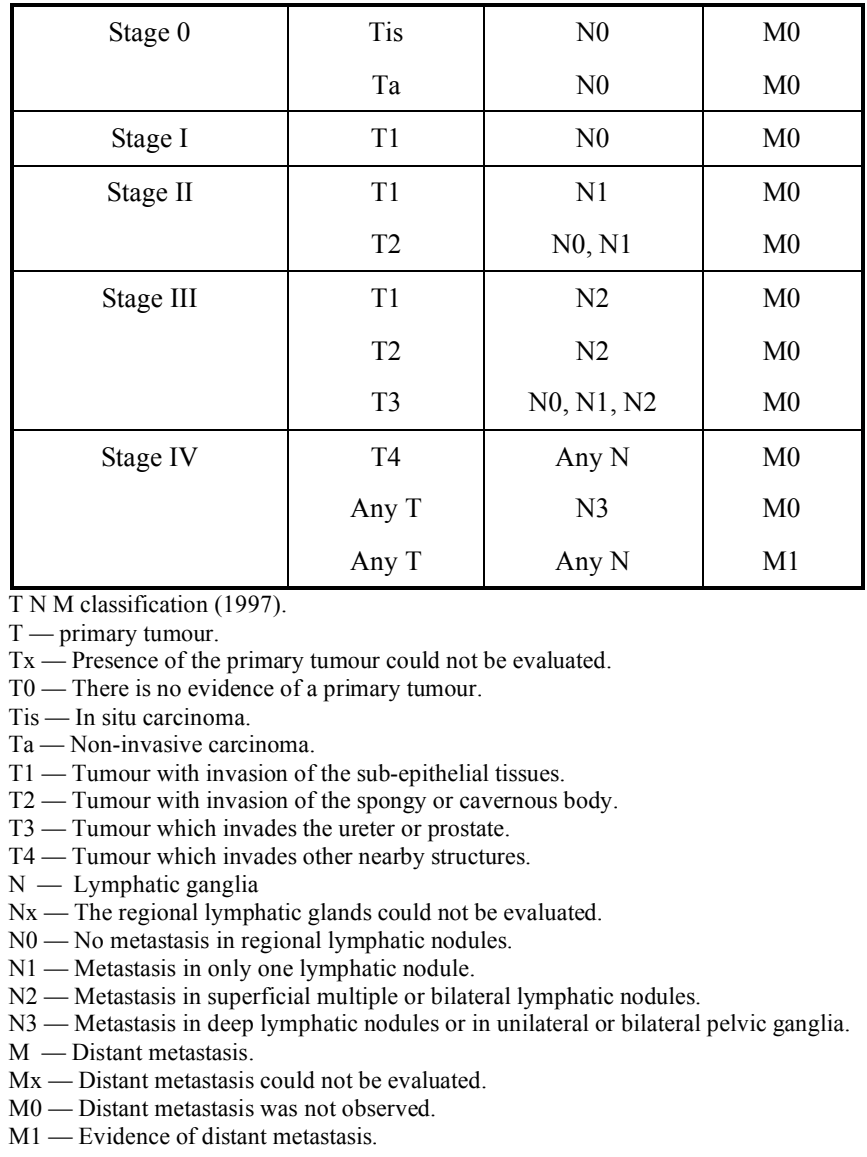

Treatment of squamous penile carcinoma could vary depending on the histological type, size and location of the lesion, tumoural stage and level of cellular differentiation [44].

\section{REFERENCES}

[1] IARC Working Group on the evaluation of the carcinogenic risks to humans. Human Papillomavirus. Monographs of the evaluation of carcinogenic risks to humans. Lyon: International Agency for Research on Cancer, World Health Organisation 1995; vol. 64.
[2] Muñoz N, Bosch FX, de Sanjose S, et al. International agency for research on cancer multicenter cervical cancer study group. Epidemiologic classification of human papillomavirus types associated with cervical cancer. N Engl J Med 2003; 348: 518-27.

[3] Walboomers JM, Jacobs MV, Manos D, et al. Human papillomavirus is a necessary cause of invasive cancer worlwide. J Pathol 1999; 189: 12-9.

[4] Mougin C, Dalstein V, Pretet JL. Epidemiology of cervical papillomavirus infections. Recent knowledge. Press Med 2001; 30: 1017-23.

[5] Trottier H, Mashmud S, Costa MC, Sobrinho JP, Duarte-Franco E, Rohan TE. Human papillomavirus infections with multiple types and risk of cervical neoplasia. Cancer Epidemiol Biomarkers Prev 2006; 15: 1274-80

[6] Rodríguez-Cerdeira C, Chillón R, Díez-Moreno S, Guerra-Tapia A. Prevalence and genotypic identification of human papillomavirus infection in a population from northwestern Spain. Open Dermatol J 2009; 3: 18-21.

[7] Rodríguez-Cerdeira C, Menéndez A, Bravo G, Alcántara R. El virus del papiloma humano y su repercusión en la patología genital femenina. Piel 2007; 22: 1171-80.

[8] Fujita M, Inoue M. Alteration of the $\mathrm{p} 53$ in human primary cervical with and without human papillomavirus infection. Cancer Res 1992; 52: 5323-8.

[9] Muller M, Gissman L, Cristiano RJ, et al. Papillomavirus capsid binding and uptake by cells from different tissues and species. J Virol 1995; 69: 948-54.

[10] Kjaer SK, van den Brule AJ, Paull G, et al. Type specific persistence of high human papillomavirus (HPV) as indicator of high grade cervical squamous intraepithelial lesions in young women, population based prospective follow up study. BMJ 2002; 325: 572-8.

[11] Castellsagué X, Díaz M, de Sanjose S, et al. Worlwide human papillomavirus etiology of cervical adenocarcinoma and its cofactors implications for screening and prevention. IARC multicenter cervical cancer study group. J Natl Cancer Inst 2006; 98: 303-15.

[12] de Sanjosé S, Almirall R, Loveras B, et al. Cervical human papillomavirus infection in the female population in Barcelona, Spain. Sex Trans Dis 2003; 30: 788-93.

[13] Rodriguez-Cerdeira C. Tumores malignos. In: Guerra TA, Ed Patología genital masculina no ITS. Monografías de Dermatología. Madrid: Grupo Aula Médica 2008; vol. 21: pp. 393-401.

[14] Rodríguez Cerdeira MC. Síntesis de controversias en Venerelogia. In: Olmos Acebes L, Ed. La consulta de ETS. Madrid: Trébol y comunicación 2005; vol. 2: pp. 62-67.

[15] Rodríguez Cerdeira C, Carpintero Feijoo Y, Alcántara Cáceres R, Vilata Corell JJ. Sífilis adquirida. In: Vilata Corell JJ, Ed. Venereología: aspectos epidemiológicos y clínicos de las infecciones transmitidas por vía sexual. Madrid: Aula Médica 2005; pp. 51-61.

[16] Castellsagué X, Díaz M, Sanjosé S. Worldwide human papillomavirus etiology of cervical adenocarcinoma and its cofactors: implications for screening and prevention. J Natl Cancer Inst 2006; 98: 303-15.

[17] Cuzick J, Sasieni P, Davies P, et al. A systematic review of the role of human papillomavirus (HPV) testing within a cervical screening programme: summary and conclusions. Br J Cancer 2000; 83: 5615.

[18] Franco EL, Durarte-Franco E, Ferenczy A. Cervical cancer: epidemiology, prevention and role of the human papillomavirus infection. CMAJ 2001; 164: 1017-25.

[19] Herrero R, Hildesheim A, Bratti C, et al. Population-based study of human papillomavirus infection and cervical neoplasia in rural Costa Rica. J Natl Cancer Inst 2000; 92: 464-74.

[20] Puig-Tintoré LM, Castellsagué X, Torné A, et al. Coverage and factors associated with cervical cancer screening: Results from the AFRODITA study, a population-based Surrey in Spain. J Low Genit Trac Dis 2008; 12: 82-9.

[21] Recomendaciones Consejo europeo. Resolución 287 de 10 de junio 2008 [Last accessed 15 May 2009]. Available at: $\mathrm{http}: / /$ www.register.consilium.europa.eu.

[22] Jordan J, Arbyn M, Martin-Hirsch P, et al. European guidelines for quality assurance in cervical cancer screening: recommendations for clinical management of abnormal cervical cytology, part 1. Cytopathology 2008; 19: 342-54. 
[23] Jordan J, Martin-Hirsch P, Arbyn M, et al. European guidelines for quality assurance in cervical cancer screening: recommendations for clinical management of abnormal cervical cytology, part 2 . Cytopathology 2009; 20: 5-16.

[24] Miranda Serrano PG, Vaquero Arguello S, Alonso García M, Osunas S. Lesiones y neoplasias del tracto genital femenino relacionadas con la infección por el virus del papiloma humano Rev Pediatr Aten Primaria 2007; 9: S43-62.

[25] Miroshnichenko GG, Parva M, Holtz DO, Klemens JA, Dunton CJ. Interpretability of excisional biopsies of the cervix: cone biopsy and loop escisión. J Low Genit Tract Dis 2009; 13: 10-2.

[26] Michy T, Leveque J, Loussouarn D, et al. Cervical pathology: significance and therapeutic impact of margin statusLa notion de marge d'exérèse dans le contexte de la pathologie du col utérin: définitions et impact thérapeutique. Bull Cancer 2008; 95: 1171-5.

[27] Jolley JA, Wing DA. Pregnancy management after cervical surgery. Curr Opin Obstet Gynecol 2008; 20: 528-33.

[28] Dunton CJ. Management of atypical glandular cells and adenocarcinoma in situ. Obstet Gynecol Clin North Am 2008; 35: 623-32.

[29] Moscicki B, Schiffman S, Kjaer S, Villa LL. Updating the natural history of HPV anogenital cancer. Vaccine 2006; 24: S42-51.

[30] Galcerán J, Marcos-Grajera R, Izquierdo A, Borrás J. Carcinoma invasor y lesiones premalignas del cuello uterino en los registros poblacionales: utilidad y limitaciones. In: Sanjosé S, García A, Eds. Virus del papiloma humano y cáncer: epidemiología y prevención. N. . 4. Barcelona: Sociedad Española de Epidemiología 2006; pp. $15-27$.

[31] Haart WR. Vulvar intraepithelial neoplasia: historical aspects and current status. Int J Gynecol Pathol 2001; 20: 16-30.

[32] de Hullu JA, Oonk NTH, van der Zee AG. Modern management of vulvar cancer. Curr Opin Obstet Gynecol 2004; 16: 65-72.

[33] Brown JV 3rd, Goldstein BH, Rettenmaier MA, Aylward MM, Graham CL, Micha JP. Laser ablation of surgical margins after excisional partial vulvectomy for VIN: effect on recurrence. $\mathrm{J}$ Reprod Med 2005; 50: 345-50.

[34] Rodríguez-Cerdeira C, Gonzalez-Guerra E, Guerra-Tapia A Cáncer de vulva. MAS Dermatologia 2008; 4: 26-32.

[35] Benedet Jl, Bender H, Hones $\mathrm{H}^{\text {rd }}$, Ngang HYS, Pecorelli S. FIGO staging classification and clinical practice guidelines in the management of gynecological cancer. FIGO comitte on gynecologic oncology. Int J Gynecol Obstet 2000; 70: 209-62.

[36] Rodríguez-Cerdeira C, de Argila D, A Guerra. Tumores malignos. Ed. Guerra Tapia A. Manual yd atlas de enfermedades de la vulva. Barcelona: Glosa 2006; pp. 261-83.

[37] Ryan DP, Willett CG. Clinical features, staging, and treatment of anal cancer. [last accessed May 2009]. Available at www.uptodate. com

[38] Palefsky J, Cranston RD. Anal squamous intraepithelial lesions (ASIL): diagnosis, screening and treatment. [last accessed 5 May 2009]. Available at: www.uptodate.com

[39] Puig-Tintoré LM, Ordi J, Torré A, Cararach M, Aymerich JP. Atlas digital de enfermedades de la vulva. Barcelona: Ėmfasi 2005.

[40] Buechner SA. Common skin disorders of the penis. BJU Int 2002; 90: 498-50.

[41] Cox NH, Eedy DJ, Morton CA. Guidelines and audit subcommittee, british association of dermatologists. guidelines for management of Bowen's disease: 2006 update. Br J Dermatol 2007; 156: 11-21.

[42] Rodríguez-Cerdeira C, de Argila Fernández-Durán, Guerra Tapia A. Tumores malignos. In: Guerra Tapia A, Ed. Manual y atlas de las enfermedades del pene. Barcelona: Editorial Glosa 2008; pp. 261-283.

[43] Hernandez BY, Barnholtz-Sloan J, German RR, et al. Burden of invasive squamous cell carcinoma of the penis in the United States, 1998-2003. Cancer 2008; 113: 2883-91.

[44] Leveridge M, Siemens DR, Morash C. What next? Managing lymph nodes in men with penile cancer. Can Urol Assoc J 2008; 2: $525-31$. 